\title{
Suppurative necrotizing granulomatous lymphadenitis in adult-onset Still's disease: a case report
}

\author{
Stelios F Assimakopoulos ${ }^{1 *}$, Vassilios Karamouzos ${ }^{1}$, Christos Papakonstantinou' ${ }^{1}$, Vassiliki Zolota ${ }^{2}$, \\ Chryssoula Labropoulou-Karatza ${ }^{1}$ and Charalambos Gogos ${ }^{1}$
}

\begin{abstract}
Introduction: Lymphadenopathy is found in about 65\% of patients with adult-onset Still's disease and is histologically characterized by an intense, paracortical immunoblastic hyperplasia. Adult-onset Still's disease has not been previously described as an etiology of suppurative necrotizing granulomatous lymphadenitis.

Case presentation: We describe a 27-year-old Greek man who manifested prolonged fever, abdominal pain, increased inflammatory markers, episodic skin rash and mesenteric lymphadenopathy histologically characterized by necrotizing granulomatous adenitis with central suppuration. Disease flares were characterized by systemic inflammatory response syndrome with immediate clinico-laboratory response to corticosteroids but the patient required prolonged administration of methylprednisolone at a dose of above $12 \mathrm{mg} /$ day for disease control. After an extensive diagnostic work-up, which ruled out any infectious, malignant, rheumatic or autoinflammatory disease the patient was diagnosed as having adult-onset Still's disease. The patient is currently treated with $4 \mathrm{mg}$ of methylprednisolone, $100 \mathrm{mg}$ of anakinra daily and methotrexate $7.5 \mathrm{mg}$ for two consecutive days per week and exerts full disease remission for six months.

Conclusion: To the best of our knowledge this is the first report of suppurative necrotizing granulomatous lymphadenitis attributed to adult-onset Still's disease. This case indicates that the finding of a suppurative necrotizing granulomatous lymphadenitis should not deter the consideration of adult-onset Still's disease as a potential diagnosis in a compatible clinical context; however, the exclusion of other diagnoses is a prerequisite.
\end{abstract}

Keywords: Adult-onset Still's disease, Granulomatous, Inflammatory, Lymphadenitis, Suppurative

\section{Introduction}

Adult-onset Still's disease (AOSD) is a systemic inflammatory disorder of unknown etiology and pathogenesis. There is no single diagnostic test for AOSD; rather, the diagnosis is based on clinical criteria, such as high daily fever, arthralgia, skin rash, lymphadenopathy, and hepatosplenomegaly, and exclusion of any infection, malignancy, or other rheumatic disorder known to mimic AOSD in its clinical features [1]. Lymphadenopathy is found in about $65 \%$ of AOSD patients and is histologically characterized by an intense, paracortical immunoblastic

\footnotetext{
* Correspondence: sassim@upatras.gr

'Department of Internal Medicine, University Hospital of Patras, Rion-Patras 26504, Greece

Full list of author information is available at the end of the article
}

hyperplasia [1-3]. To the best of our knowledge, findings of suppurative necrotizing granulomatous lymphadenitis have not been previously described in AOSD.

The differential diagnosis of a necrotizing granulomatous lymphadenitis is wide, including: infectious diseases (bacterial, viral, fungal or parasitic); malignant disorders, mainly lymphoid malignancies; autoimmune disorders like systemic lupus erythematosus; autoinflammatory diseases; and idiopathic causes like Kikuchi's disease and sarcoidosis [4-6]. The presence of variable degrees of suppuration although being suggestive of specific diagnoses like Yersinia pseudotuberculosis infection or tuberculosis (TB) does not preclude other potential causes of necrotizing granulomatous adenitis [4].

\section{Biomed Central}

(c) 2012 Assimakopoulos et al.; licensee BioMed Central Ltd. This is an Open Access article distributed under the terms of the Creative Commons Attribution License (http://creativecommons.org/licenses/by/2.0), which permits unrestricted use, distribution, and reproduction in any medium, provided the original work is properly cited. 
Here we describe a case of suppurative necrotizing granulomatous lymphadenitis attributed to AOSD after an extensive diagnostic work-up, which ruled out all the aforementioned etiologies.

\section{Case presentation}

A 27-year-old Greek man, with an unremarkable past medical history except a reported penicillin allergy, was admitted to our department, referred from a regional hospital, for investigation of fever of three months' duration associated with mesenteric lymphadenopathy, leukocytosis (25.000/ $\mathrm{mm}^{3}$ with polymorphonuclear predominance) and increased erythrocyte sedimentation rate (ESR) over $100 \mathrm{~mm} / \mathrm{h}$. The patient, who reported no close animal contact, had been previously hospitalized twice in the referral hospital complaining of fever associated with rigors and right lower guardant abdominal pain. The performed diagnostic investigation had only shown mesenteric lymphadenopathy and the patient was conservatively treated with intravenous antibiotic therapy consisting of ciprofloxacin and metronidazole with temporal improvement, followed by relapse after a short time.

On admission, the patient's temperature was $39^{\circ} \mathrm{C}$, heart rate was $90 \mathrm{bpm}$, respiratory rate was 16 breaths per minute, blood pressure was $120 / 80 \mathrm{mmHg}$ and oxygen saturation in room air was $97 \%$. His physical examination was unremarkable except for abdominal tenderness in his right lower guardant without rebound tenderness. No hepatomegaly, splenomegaly or peripheral lymphadenopathy was detected.

An initial laboratory evaluation showed a white blood cell count of $22.3 \times 10^{9} / \mathrm{L}$, with predominant neutrophils (80\%), hematocrit 35\%, hemoglobin $11.6 \mathrm{~g} / \mathrm{dL}$ (mean corpuscular volume $=82$ and mean corpuscular hemoglobin =26) and platelet count $51 \times 10^{9} / \mathrm{L}$. Prothrombin and partial thromboplastin times were normal and d-dimers were slightly increased at $0.72 \mu \mathrm{g} / \mathrm{ml}$. The blood biochemistries, including serum angiotensin-converting enzyme (ACE) and protein electrophoresis were all normal. Serum C-reactive protein was increased at $17.3 \mathrm{mg} / \mathrm{dL}$, ESR at $80 \mathrm{~mm} / \mathrm{h}$, fibrinogen at $851 \mathrm{mg} / \mathrm{dL}$ and ferritin at $663 \mathrm{mg} / \mathrm{dL}$. Thyroid function tests were normal. Urine analysis and 24-hour urinary calcium and protein excretion were normal. Electrocardiogram, chest X-ray, echocardiogram and arterial blood gas were also normal. A detailed ophthalmologic examination including slit-lamp eye examination, fundoscopy, Rose Bengal test and Schirmer's test was unrevealing.

An abdominal ultrasonography revealed enlarged mesenteric lymph nodes of $2.5 \mathrm{~cm}$ diameter. Consequent thoracic and abdominal computed tomography (CT) scans confirmed the presence of mesenteric lymphadenopathy of $2.7 \mathrm{~cm}$ maximum diameter with an hypodense center (Figure 1A, 1B), without evidence of bowel inflammation
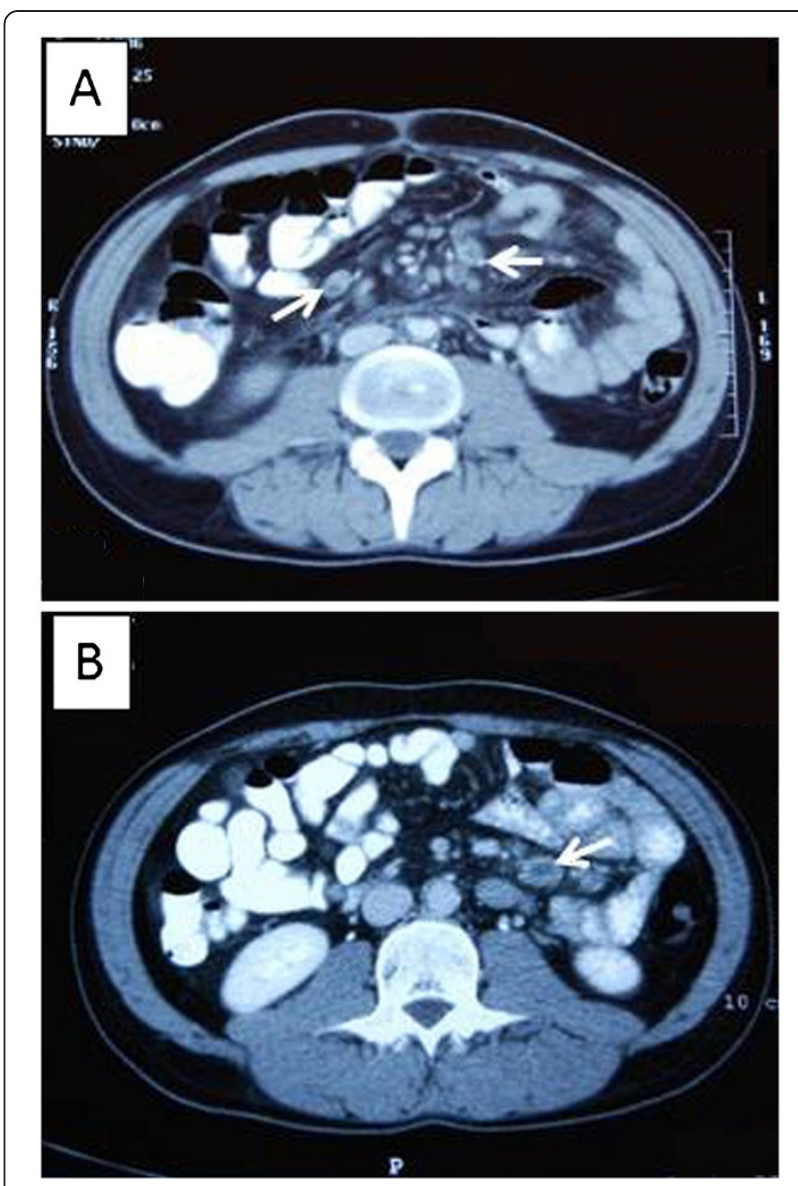

Figure 1 Computed tomography scan of the patient's abdomen (A and B): A mesenteric lymphadenopathy of $2.7 \mathrm{~cm}$ maximum diameter with a hypodense center was detected (white arrows).

or hepatosplenomegaly, with normal vascular perfusion of abdominal organs, absence of mediastinal lymphadenopathy, ascites, pleural or pericardial effusions.

All sets of blood cultures (at least six), urine and stool cultures and examination for ova and parasites were negative. Antibodies for hepatitis A, B, C, coxsackie, Enteric Cytopathic Human Orphan virus, herpes simplex virus, Epstein-Barr virus, cytomegalovirus, human immunodeficiency virus, human T-lymphotropic virus-1 and virus-2, Yersinia enterocolitica and Entamoeba histolytica, Bartonella henselae, Francisella tularensis, Leishmania donovani, Coxiella burnetii and Rickettsia conorii were negative. Wright and rapid plasma reagin tests were also negative, whereas the tuberculin skin test was positive (15mm).

The patient underwent upper and lower gastrointestinal tract endoscopies, enteroclysis and capsule endoscopy without evidence of inflammatory bowel disease, infective colitis or celiac disease. Examination of gastric fluid with Ziehl-Neelsen stain detected no acid-fast bacteria; a 
polymerase chain reaction (PCR) as well as culture for mycobacterium TB were negative. Small bowel (jejunal and ileal) and colonic biopsies presented findings of nonspecific inflammatory reaction, the architecture of intestinal villi was preserved and mucosal periodic acid-Schiff stain for detection of Tropheryma whipplei was negative.

A full immunologic screening with rheumatoid factor, antinuclear antibodies, antibodies to double-stranded DNA, anti-Sm, anti-Ro/SSA, anti-La/SSB, anti-RNP, anti-Jo-1, anti-Scl-70, anti-histones, anti-mitochondrial antibodies, anti-smooth muscle antibodies, cytoplasmic-antineutrophil cytoplasmic antibody (ANCA), perinuclear-ANCA, antitransglutaminase, anti-cardiolipin, and lupus anticoagulant was negative. Serum complement and levels of immunoglobulins (Igs, IgA, IgG, IgM, IgE, IgD) were normal. The results of genetic testing for mutation of the familial Mediterranean fever gene (M694V, V726A, M694I, M680I, and E148Q) were negative. In addition, the results of a full tumor marker profile were also normal.

The patient underwent laparotomy with mesenteric lymph node excision for microbiological and histological examination. Histology revealed a granulomatous lymphadenitis with central suppurative necrosis (Figure 2A). Gram, Giemsa, Ziehl-Neelsen and Grocott methenamine silver stains for detection of common bacteria, mycobacteria and fungi were all negative. Immunohistochemical studies for lymphoproliferative disease were unrevealing. In addition, the patient's lymph node was examined with a PCR and cultures for mycobacterium TB, atypical mycobacteria (Mycobacterium africanum I and/or II, M. microti, M. carnetti, M. bovis, and M. avium complex), fungi and Tropheryma whipplei without detection of any pathogen. A gastrocnemius muscle biopsy was

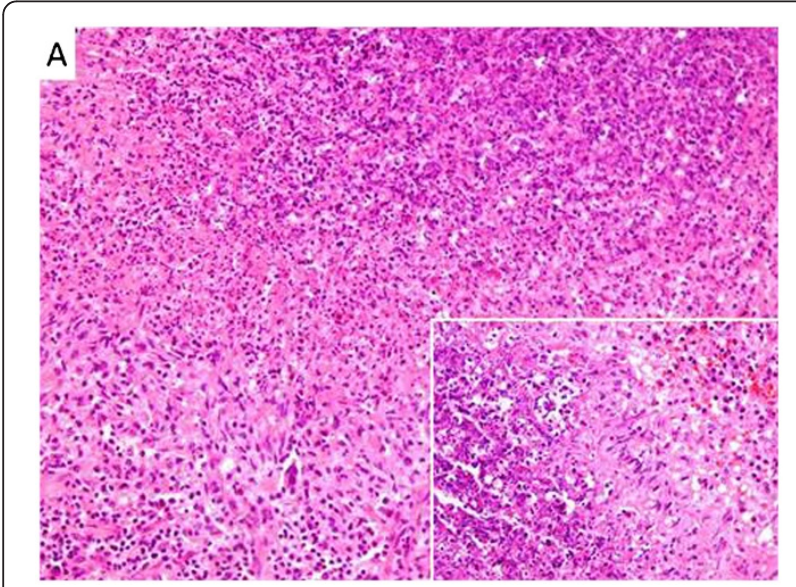

Figure 2 Mesenteric lymph node biopsy from the presented patient: formation of histiocytic granulomas with central suppurative necrosis (hematoxylin and eosin stain, $A: \times 100$, insert $\times 200$ ). also performed without identification of sarcoid granulomata.

The hematological work-up in the investigation of the patient included microscopic examination of peripheral blood film which showed no evidence of lymphoproliferative disorders. A bone marrow biopsy and immunophenotypic analysis revealed no pathologic findings. A peripheral blood immunophenotypic analysis revealed lymphopenia due to simultaneous decrease of B-, T- and NK-cells. Serum beta-2-microglobulin levels were within normal limits.

After an extensive diagnostic work-up no definite diagnosis was available for our patient. During hospitalization the patient was empirically treated with combined antimicrobial therapy that consisted of ciprofloxacin and metronidazole without response, while episodic disease flares characterized by a systemic inflammatory response syndrome (SIRS) and spikes of increased markers of inflammation were recorded. Sporadically, fever spikes were associated with a transient maculopapular skin rash in his upper extremities. Of note, the patient experienced a dramatic clinical improvement after administration of corticosteroids for a short time; the corticosteroids were administered due to his history of allergy in order to administer intravenous contrast media for CT performance. This temporal improvement was shortly followed by disease relapse. Based on the therapeutic criterion of response to corticosteroids and taking into consideration the significantly positive tuberculin skin test in conjunction with the histology of the mesenteric lymph node, which showed a granulomatous necrotizing lymphadenitis with central suppuration, we decided to administer a combination empiric therapy. The therapy consisted of corticosteroids (methylprednisolone 16mg) and anti-TB drugs (isoniazide $150 \mathrm{mg} / \mathrm{d}$, pyrazinamide $30 \mathrm{mg} / \mathrm{kg}$, ethambutol $20 \mathrm{mg} / \mathrm{kg}$ and moxifloxacin $400 \mathrm{mg} / \mathrm{d}$ ). Rifampicin $300 \mathrm{mg} / \mathrm{d}$, which was included in our initial scheme, was later discontinued due to induction of rifampicin-associated pancreatitis. He received the anti-TB therapy for one year and discontinued corticosteroids after a very slow tapering at 11 months. During this period the patient was asymptomatic with normal values of inflammatory markers and he had a total resolution of mesenteric lymphadenopathy at abdominal $\mathrm{CT}$ re-examination on completion of the anti-TB therapy. Unfortunately, 20 days after stopping the corticosteroids the disease relapsed, and the patient had fever, abdominal pain, increased markers of inflammation and reappearance of mesenteric lymphadenopathy on abdominal MRI. The patient's symptoms were controlled with $16 \mathrm{mg}$ methylprednisolone; however, while attempting to taper the methylprednisolone to $12 \mathrm{mg}$ after about two months of treatment, his symptoms relapsed forcing us to reinstitute a higher 
Table 1 Causes of granulomatous and/or necrotizing lymphadenitis, with or without suppuration

Granulomas Necrosis Suppuration

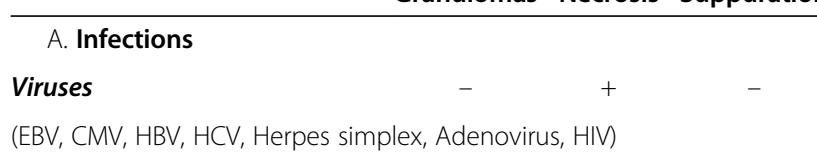

\section{Bacteria}

Yersinia pseudotuberculosis

Bartonella

Tropheryma whipplei

Francisella tularensis

Brucella

Spirochaetes

Treponema pallidum

Chlamydia

Lymphogranuloma

venereum

Rickettsia

Coxiella burnetii

Mycobacteria

Mycobacterium tuberculosis

Atypical mycobacterial

infection

Mycobacterium leprae

Parasites

Toxoplasmosis

Leishmaniasis

Fungi

Histoplasmosis

Aspergillosis

Coccidioidomycosis

Cryptococcosis

B. Neoplastic diseases

Hodgkin disease

Non-Hodgkin disease

Metastatic carcinoma

Langerhans cell histiocytosis

Seminoma

Dysgerminoma

C. Autoimmune diseases

Systemic lupus erythematosus

Granulomatosis with polyangiitis

Churg-Strauss syndrome

Celiac disease

Crohn's disease

Primary biliary cirrhosis

Kawasaki disease

Table 1 Causes of granulomatous and/or necrotizing lymphadenitis, with or without suppuration (Continued)

D. Idiopathic diseases

Sarcoidosis

Necrotic sarcoid granulomatosis

Kikuchi-Fujimoto disease

E. Autoinflammatory diseases

Familial Mediterranean fever

Hyperimmunoglobulinemia-D no data no data no data syndrome

PFAPA syndrome

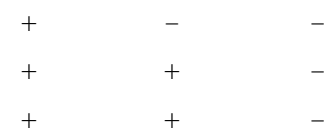

$\begin{array}{ccc}- & + & - \\ \text { no data } & \text { no data } & \text { no data }\end{array}$

Note: \pm indicates the potential existence of the corresponding histological feature, which is however non-typical for this disease.

CMV, Cytomegalovirus; EBV, Epstein-Barr virus; HBV, Hepatitis B; HCV, Hepatitis C; HIV, Human immunodeficiency virus; PFAPA, Periodic fever, aphthous stomatitis, pharyngitis and adenitis.

corticosteroid dose. Our second effort to gradually reduce the corticosteroid dose, after control of the patient's symptoms and total remission of mesenteric lymphadenopathy on abdominal MRI, led to disease relapse when the dose of methylprednisolone was reduced to $12 \mathrm{mg} /$ day. Disease relapse was controlled by increasing methylprednisolone dose to $16 \mathrm{mg}$, while additional immunomodulatory drugs (anakinra and methotrexate) were added in order to prevent disease relapse during methylprednisolone tapering. The patient is currently treated with $4 \mathrm{mg}$ of methylprednisolone, $100 \mathrm{mg}$ of anakinra daily and methotrexate $7.5 \mathrm{mg}$ for two consecutive days per week and has experienced full disease remission for six months.

\section{Discussion}

Here we describe a previously healthy young man who presented with prolonged fever, abdominal pain, increased inflammatory markers, episodic skin rash and mesenteric lymphadenopathy histologically characterized by necrotizing granulomatous adenitis with central suppuration. Disease flares were characterized by SIRS with immediate clinico-laboratory response to corticosteroids but the patient required prolonged administration of methylprednisolone at a dose of above $12 \mathrm{mg} /$ day for disease control.

In Table 1 we present an extensive differential diagnosis of granulomatous necrotizing and suppurative lymphadenitis $[4,5,7]$. As shown in Table 1, histological overlapping of the formation, necrosis and suppuration of granulomas, in variable degrees, may exist in diverse lymphadenopathies. Therefore, when a suppurative necrotizing granulomatous lymphadenitis cannot be associated with any of its typical causes, our diagnostic consideration should encompass all the potential causes of granulomatous and necrotizing lymphadenitis. 
In our patient, no infectious etiology could be detected by serological, microbiological, histological and molecular methods. Whipple's disease, which was a serious diagnostic consideration, was thoroughly investigated and excluded. Lymph node histology in conjunction with positive Mantoux reaction, compatible clinical picture, and absence of an alternative confirmed diagnosis led to institution of empiric anti-TB therapy. Disease relapse after completion of one year's anti-TB therapy was the strongest criterion for exclusion of tuberculous lymphadenitis.

Lymphoid malignancies, either non-Hodgkin or Hodgkin disease, were excluded based on lymph node histologic and immunohistochemical studies, bone marrow aspiration, biopsy and immunophenotypic analysis, and peripheral blood smear examination and immunophenotypic analysis.

Crohn's disease was another important diagnostic possibility. Normal upper and lower gastrointestinal tract endoscopies, enteroclysis, capsule endoscopy and small and large intestinal biopsies excluded this diagnosis.

Sarcoidosis was also considered to be a diagnostic possibility in our patient. The typical histological feature of sarcoidosis is the formation of non-necrotizing granulomata [8]; however, the existence of necrotizing sarcoid granulomatosis has also been described since 1973 [9]. Normal serum ACE levels, absence of hypercalciuria, normal pulmonary function tests, slit-lamp eye examination, CD4 to CD8 ratio in bronchoalveolar lavage, and no detection of sarcoid granulomata in gastrocnemius muscle biopsy [10], made this diagnosis unlikely.

Regarding autoimmune diseases, systemic lupus erythematosus was excluded by the absence of autoantibodies. The possibility of granulomatosis with polyangiitis and Churg-Strauss syndrome was ruled out based mainly on clinical criteria combined with negative ANCAs. Kawasaki disease, which occurs rarely in adults, was excluded by incompatible clinical course because it is typically a self-limited condition, while its associated necrotizing lymphadenitis is typically non-granulomatous [11].

Kikuchi-Fujimoto disease was excluded based on the prolonged and relapsing clinical course of our patient and the histological features of affected lymph nodes with significant infiltration by neutrophils [12]. Among autoinflammatory diseases, the familial Mediterranean fever was excluded by appropriate genetic testing, the hyper-IgD syndrome by normal serum $\operatorname{IgD}$ and $\operatorname{IgA}$ levels and periodic fever with aphthous stomatitis, pharyngitis, and adenitis syndrome by clinical criteria [13].

After the aforementioned extensive diagnostic workup and ruling out all potential alternative diagnostic considerations, AOSD, an inflammatory disorder which could be expressed with daily fevers, increased inflammatory markers and lymphadenopathy, in the absence of positive autoantibodies, came into play as an important diagnostic consideration. Disease presentation in the patient was atypical for AOSD because it lacked common features such as arthralgias, sore throat, abnormal liver function tests and significant serum hyperferritinemia [14]. However, diagnosis of AOSD was based on fulfillment of the commonly used high sensitive Yamaguchi criteria (93.5\% sensitivity) and exclusion of any other diagnostic consideration including any infectious, malignant, or rheumatic disorder known to mimic AOSD in its clinical features (Table 2) [1,15]. Although a lymph node biopsy is not necessary for the diagnosis of AOSD it is often performed to rule out other potential diagnostic thoughts, like lymphomas. Lymphadenopathy in AOSD patients is histologically characterized by an intense, paracortical immunoblastic hyperplasia, whereas the finding of a suppurative necrotizing granulomatous lymphadenitis has not been previously described [1-3].

\section{Conclusion}

To the best of our knowledge this is the first report of suppurative necrotizing granulomatous lymphadenitis attributed to AOSD. The presented case demonstrates that the finding of a suppurative necrotizing granulomatous lymphadenitis should not deter the consideration of

Table 2 Accordance of the presented patient with the Yamaguchi criteria for the diagnosis of adult-onset Still's disease

\begin{tabular}{lc}
\hline $\begin{array}{l}\text { Yamaguchi criteria (require the presence } \\
\text { of five features, with at least two being } \\
\text { major diagnostic criteria): }\end{array}$ & $\begin{array}{c}\text { Patient's } \\
\text { characteristics }\end{array}$ \\
\hline Major Yamaguchi criteria: & + \\
1. Fever of at least $39^{\circ} \mathrm{C}$ lasting at least one week. & - \\
2. Arthralgias or arthritis lasting two weeks or longer. & + \\
3. Typical rash (maculopapular, nonpruritic) & + \\
during febrile episodes. & \\
4. Leukocytosis (10,000/ $\mu$ L or greater), & - \\
with at least $80 \%$ granulocytes. & + \\
Minor Yamaguchi criteria: & - \\
1. Sore throat & - \\
2. Lymphadenopathy & + \\
3. Hepatomegaly or splenomegaly & \\
4. Abnormal liver function studies & \\
5. Negative antinuclear antibodies & \\
and rheumatoid factor. & \\
Exclusions & \\
1. Infection, especially sepsis and infectious & \\
mononucleosis & \\
2. Malignancies, especially lymphomas & \\
3. Rheumatic diseases known to mimic adult & \\
Still's disease & \\
\hline
\end{tabular}


AOSD as a potential diagnosis when the clinical features are compatible; however, exclusion of a wide range of infections, malignancies and other rheumatologic conditions should always be performed.

\section{Consent}

Written informed consent was obtained from the patient for publication of this case report and accompanying images. A copy of the written consent is available for review by the Editor-in-Chief of this journal.

\section{Abbreviations}

ACE: angiotensin-converting enzyme; ANCA: antineutrophil cytoplasmic antibody; AOSD: Adult-onset Still's disease; CT: computed tomography; ESR: erythrocyte sedimentation rate; Ig: immunoglobulin, PCR, polymerase chain reaction; SIRS: systemic inflammatory response syndrome;

TB: tuberculosis.

\section{Competing interests}

The authors declare that they have no competing interests.

\section{Authors' contributions}

SFA wrote this case report; SFA, VK and CP, were the patient's doctors; VZ performed the histological diagnosis; CL-K and CG critically revised the manuscript. All authors have read and approved the final version of this manuscript.

\section{Author details}

${ }^{1}$ Department of Internal Medicine, University Hospital of Patras, Rion-Patras 26504, Greece. ²Department of Pathology, University Hospital of Patras, Rion-Patras 26504, Greece.

Received: 25 June 2012 Accepted: 19 September 2012

Published: 18 October 2012

\section{References}

1. Kadar J, Petrovicz E: Adult-onset Still's disease. Best Pract Res Clin Rheumatol 2004, 18:663-676.

2. Valente RM, Banks PM, Conn DL: Characterization of lymph node histology in adult onset Still's disease. J Rheumatol 1989, 16:349-354.

3. Quaini F, Manganelli P, Pileri S, Magnani G, Ferrari C, Delsignore R, Sabattini E, Olivetti G: Immunohistological characterization of lymph nodes in two cases of adult onset Still's disease. J Rheumatol 1991, 18:1418-1423.

4. James DG: A clinicopathological classification of granulomatous disorders. Postgrad Med J 2000, 76:457-465.

5. Dorfman RF, Berry GJ: Kikuchi's histiocytic necrotizing lymphadenitis: an analysis of 108 cases with emphasis on differential diagnosis. Semin Diagn Pathol 1988, 5:329-345.

6. Zumla A, James DG: Granulomatous infections: etiology and classification. Clin Infect Dis 1996, 23:146-158.

7. Fraser IP: Suppurative lymphadenitis. Curr Infect Dis Rep 2009, 11:383-388.

8. Newman LS, Rose CS, Maier LA: Sarcoidosis. N Engl J Med 1997, 336:1224-1234.

9. Liebow AA: The J. Burns Amberson lecture-pulmonary angiitis and granulomatosis. Am Rev Respir Dis 1973, 108:1-18.

10. Andonopoulos AP, Papadimitriou C, Melachrinou M, Meimaris N, Vlahanastasi C, Bounas A, Georgiou P: Asymptomatic gastrocnemius muscle biopsy: an extremely sensitive and specific test in the pathologic confirmation of sarcoidosis presenting with hilar adenopathy. Clin Exp Rheumatol 2001, 19:569-572.

11. Burns JC, Glode MP: Kawasaki syndrome. Lancet 2004, 364:533-544.

12. Hutchinson CB, Wang E: Kikuchi-Fujimoto disease. Arch Pathol Lab Med 2010, 134:289-293.

13. Samuels J, Ozen S: Familial Mediterranean fever and the other autoinflammatory syndromes: evaluation of the patient with recurrent fever. Curr Opin Rheumatol 2006, 18:108-117.
14. Lian F, Wang Y, Yang $X, X u H$, Liang L: Clinical features and hyperferritinemia diagnostic cutoff points for AOSD based on ROC curve: a Chinese experience. Rheumatol Int 2012, 32:189-192.

15. Masson C, Le Loet X, Liote F, Dubost JJ, Boissier MC, Perroux-Goumy L, Bregeon C, Audran M: Comparative study of 6 types of criteria in adult Still's disease. J Rheumatol 1996, 23:495-497.

doi:10.1186/1752-1947-6-354

Cite this article as: Assimakopoulos et al.: Suppurative necrotizing granulomatous lymphadenitis in adult-onset Still's disease: a case report. Journal of Medical Case Reports 2012 6:354.

\section{Submit your next manuscript to BioMed Central and take full advantage of:}

- Convenient online submission

- Thorough peer review

- No space constraints or color figure charges

- Immediate publication on acceptance

- Inclusion in PubMed, CAS, Scopus and Google Scholar

- Research which is freely available for redistribution 